\title{
ANÁLISE COMPARATIVA DO COMPORTAMENTO DA ESTRUTURA DE CONTENÇÃO DO TIPO TERRA ARMADA CONSIDERANDO A MODIFICAÇÃO DO MATERIAL PREVISTO EM PROJETO.
}

\section{COMPARATIVE ANALYSIS OF THE BEHAVIOR OF THE REINFORCED SOIL CONTAINMENT STRUCTURE CONSIDERING THE MODIFICATION OF THE MATERIAL EXPECTED IN PROJECT.}

\section{Bruno César de Castro Cardoso; Beatriz de Mello Massimino.}

Universidade do Oeste Paulista - UNOESTE, Curso de Engenharia Civil, Presidente Prudente, SP

E-mail: brunocardoso@outlook.com.br, beatrizmassimino@gmail.com

RESUMO - A execução de obras na área da geotecnia sucedem a uma série de cálculos e análises preliminares, que por sua vez devem ser observados afim de preservar a integridade física do empreendimento e a segurança das pessoas que o irão utilizar. Alterações não previstas em projeto, por mais que possam vir a contribuir para a execução do mesmo e melhoria do resultado final, ocorrem muitas vezes sem o conhecimento do autor, resultando em não conformidades de projeto. Neste sentido, este trabalho desenvolve uma análise comparativa entre o projeto calculado e dimensionado de uma estrutura de contenção e o projeto executado, onde foram integralmente substituídos os materiais de composição do maciço previstos. Para isso, foram calculados os fatores de segurança ao tombamento e ao deslizamento das referidas estruturas através do método da Teoria de Rankine, posteriormente, os resultados foram dispostos em tabelas para a melhor apreciação.

Palavras-chave: Terra Armada, Contenção, Compactação, BGS, Não Conformidade.

ABSTRACT - The execution of works in the area of geotechnics follow a series of calculations and preliminary analyzes, which in turn must be observed in order to preserve the physical integrity of the enterprise and the safety of the people who will use it. Changes not foreseen in the project, however much they may contribute to its execution and improvement of the final result, occur often without the author's knowledge, resulting in non-conformities of the project. In this sense, this work develops a comparative analysis between the calculated and dimensioned design of a containment structure and the executed project, in which the planned mass composition materials were

Recebido em: $17 / 08 / 2018$ Revisado em: 12/09/2018 Aprovado em: 19/09/2018 completely replaced. For this, the safety factors were calculated to the tipping and the sliding of said structures through the method of Rankine's Theory, later, the results were arranged in tables for the best appreciation.

Keywords: Armed Earth, Containment, Compaction, BGS, Non Conformity. 


\section{INTRODUÇÃO}

Casos de falhas em estruturas de contenção devido a falhas de projeto ainda são muito recorrentes na realidade da construção civil. De maneira geral, falhas no dimensionamento, como subestimação de fatores como valores potenciais de empuxo, não observância de nível de água na camada, utilização de dados, para cálculos, de materiais de difícil acesso forçando a alteração do material na execução do projeto, são, assim como as falhas de execução, grandes causadores de sinistros nesse tipo de estrutura.

Segundo Mayr (2000), pode-se dizer que o projeto está em conformidade com a execução quando o objeto construído corresponde fielmente ao objeto projetado.

As estruturas de contenção apresentam soluções técnicas de engenharia de grandes magnitudes, de maneira que cada escolha no momento do dimensionamento de um projeto como este deve ser acompanhado de um estudo de caso elaborado e com dados reais relativos às características dos materiais e do local onde será constituída.

O objetivo geral deste trabalho é desenvolver uma análise técnica comparativa de desempenho entre o projeto calculado e dimensionado de uma estrutura de contenção caracterizada por um muro de terra armada e o projeto executado, onde foram integralmente substituídos os materiais de composição do maciço previstos em projeto por outro material de acesso facilitado no local da execução, resultando em uma execução menos complicada do ponto de vista logístico.

Os objetivos específicos deste trabalho são: realizar cálculo dos empuxos e fator de segurança ao tombamento e deslizamento do maciço para o material utilizado na execução da contenção e para o material utilizado no projeto $\mathrm{e}$ dimensionamento, discutir sobre possíveis falhas oriundas da substituição do material e concluir sobre as consequências da substituição do material utilizado no maciço.

\section{REVISÃO BIBLIOGRÁFICA}

\subsection{Falhas de Projeto e Não Conformidades}

Embora o projeto seja uma parte primordial para o sucesso da obtenção do objeto a ser construído, no Brasil ele, muitas vezes, é tomado como uma formalidade a ser vencida na pretensão da obtenção das licenças cabíveis para que haja o início da obra. (CORREIA et al., 2017)

Por sua vez, o projeto quando mal definido, incompleto ou que não contemple de maneira segura a solução que fora adotada para aquele caso, pode acarretar uma sucessão de tomadas de decisão durante o andamento da obra, que por sua vez tem como características marcantes serem decisões apressadas e pontuais, não levando em consideração as demais etapas do projeto e muitas vezes abstraindo as considerações de cálculo utilizadas no projeto. (MAYR, 2000)

Segundo Mayr (2000), estas decisões, por mais que elas possam de algum modo virem a contribuir para a execução do projeto e melhoria do resultado final, ocorrem muitas vezes sem o devido conhecimento e o consentimento do autor do projeto, levando assim, à não conformidade do projeto executado com o projeto calculado. Tais não conformidades culminam na perda de conteúdos formais do projeto, como memorias de cálculo, projeto arquitetônico, projeto estrutural, que poderão e deverão ser consultados a qualquer momento caso haja um sinistro envolvendo o mesmo.

\subsection{Estruturas de Contenção em Terra Armada}

O tipo de contenção denominado de Terra Armada ou Solo Armado consiste em um maciço de solo ou rocha reforçado com elementos inextensíveis, como vergalhões de aço ou fitas, que podem ou não ser nervuradas, podendo ser metálicas ou não, 
ligados a placas de concreto armado préfabricadas denominadas de escamas ou elementos de pele. Como podemos facilmente identificar na figura 1. Estes tipos de soluções geotécnicas, por terem uma capacidade de suportar carregamentos extremamente alta, são amplamente empregados em locais onde se tem a necessidade de contenções altas e que estão sujeitas à carregamentos excepcionais. (MAGALHÃES e AZEVEDO, 2016 )

Figura 1. Esquema de um muro de Terra Armada.

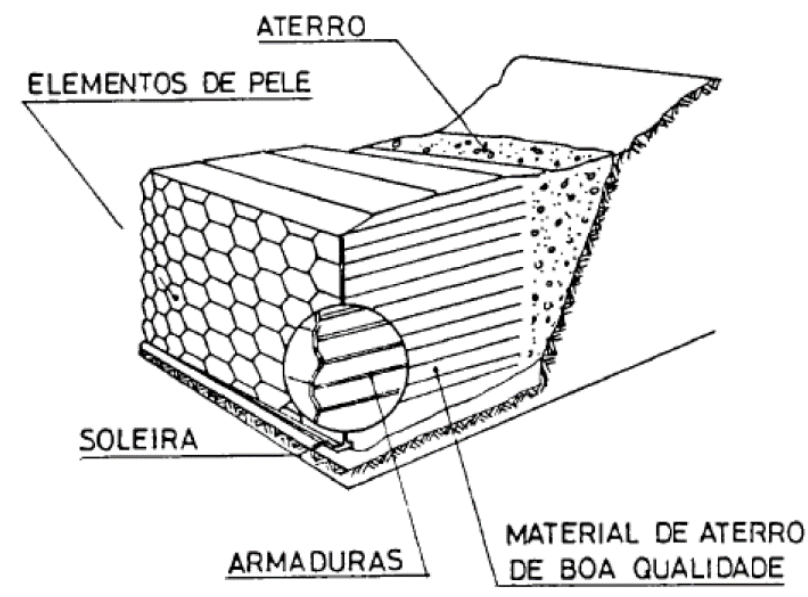

Fonte: (SILVA, 2012)

\section{MÉTODOS DE DIMENSIONAMENTO EMPREGADOS PARA ANÁLISE}

O dimensionamento dos muros de Terra Armada é composto pelos cálculos da estabilidade externa e estabilidade interna. $A$ estabilidade externa é composta por deslizamento da base da estrutura reforçada, tombamento em torno do pé do muro, ruptura do solo de fundação e ruptura global por uma superfície envolvendo todo o aterro reforçado, conforme a Figura 2. (BENJAMIM, 2006)

Figura 2. Modos de ruptura na análise da estabilidade externa.

Fonte: (BENJAMIM, 2006)
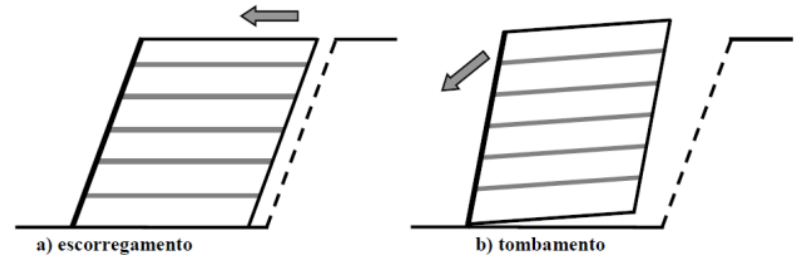

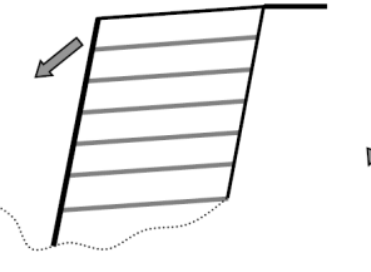

c) Capacidade de carga do solo de fundação

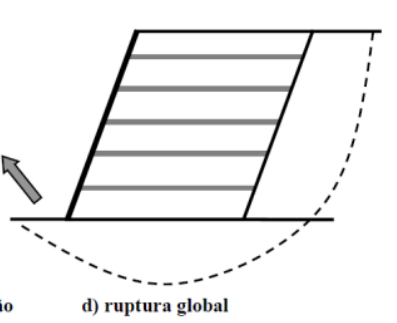

quanto ao tombamento e deslizamento das estruturas de contenção em aterro armado

Para verificação da estabilidade 
que é mais conservador em relação aos métodos utilizados, resultando em valores de empuxo maiores e, consequentemente, um dimensionamento do lado da segurança.

Rankine trabalha com duas hipóteses simplificadoras, a que os estados plásticos se desenvolvem por completo em toda a massa de solo, caracterizando perfeitamente as superfícies de ruptura como superfícies plana, e a de que o tardoz, que é a área de contato do muro com o solo, seja liso, ou seja, o atrito entre o solo e o muro é nulo. Tais hipóteses implicam em empuxos horizontalizados, quando o terreno for horizontal, e verticais, quando o terreno for vertical.

\subsection{Estabilidade externa}

$\mathrm{Na}$ estabilidade externa, foram consideradas as análises de segurança ao deslizamento, tombamento.

\subsection{Seção do Muro em Estudo}

Para realizar a comparação entre as soluções utilizadas na execução da contenção analisada, foi padronizado a geometria da contenção, com o intuito de isolar o fator "parâmetros do solo" no qual se baseia o objeto de análise deste estudo. O desenho técnico da seção do muro de contenção apresentado na Figura 3 representa as dimensões reais encontradas in-loco.

Figura 3. Seção adotada para estudo.

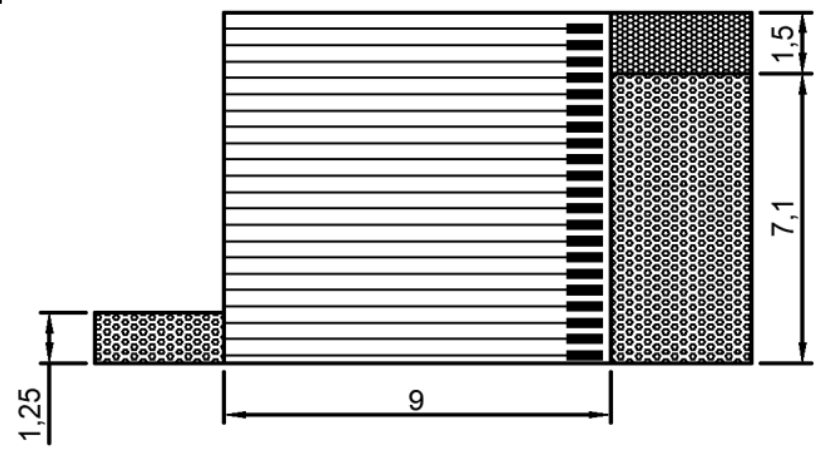

Fonte: Os autores.

O valor da sobrecarga (q) exercida pela rodovia foi retirado do memorial de cálculo do projeto, correspondendo a 2 $\mathrm{tf} / \mathrm{m}^{2}$.

\subsection{Obtenção dos Parâmetros dos Materiais para Cálculo dos Empuxos e Fatores de Segurança}

Para efeito de cálculo, os dados dos materiais utilizados no dimensionamento da estrutura de contenção foram retirados do projeto executivo do mesmo, entretanto, estes foram ensaiados em laboratório afim de assegurar e ratificar os dados contidos no documento
Já para a obtenção dos dados necessários para a realização dos cálculos dos empuxos e dos fatores de segurança levando em conta a troca de material, se fez necessário a coleta de material no local da construção do muro, bem como ensaios laboratoriais para a determinação dos parâmetros necessários para os cálculos, uma vez que nos documentos pertinentes não existiam tais dados.

Os índices físicos dos materiais que seriam utilizados como aterro e base e maciço estão contidos na figura 4 abaixo: 
Figura 4. Índices Físicos dos Materiais Considerados em Projeto.

\begin{tabular}{|c|c|}
\hline \multicolumn{2}{|c|}{ Parâmetros do Solo Aterro } \\
\hline Material 1 & Solo \\
\hline$\varnothing$ & $18^{\circ}$ \\
\hline $\mathrm{c}\left(\mathrm{tf} / \mathrm{m}^{2}\right)$ & 2,0 \\
\hline$\left.\Upsilon \mathrm{(tf} / \mathrm{m}^{3}\right)$ & 1,7 \\
\hline
\end{tabular}

\begin{tabular}{|c|c|}
\hline \multicolumn{2}{|c|}{ Parâmetros do Solo Base } \\
\hline Material 2 & Solo \\
\hline$\varnothing$ & $30^{\circ}$ \\
\hline $\mathrm{c}\left(\mathrm{tf} / \mathrm{m}^{2}\right)$ & 0,5 \\
\hline$\Upsilon\left(\mathrm{tf} / \mathrm{m}^{3}\right)$ & 1,7 \\
\hline
\end{tabular}

Fonte: (Empresa Executora da Obra)

\section{CÁLCULO DOS EMPUXOS E FATOR DE SEGURANÇA AO TOMBAMENTO E DESLIZAMENTO}

\subsection{Coeficiente de Empuxo Ativo}

Coeficiente de empuxo ativo é o esforço devido a pressão do solo sobre o muro, em razão da deformação do muro no sentido contrário ao terrapleno causando assim tração no solo. Este é definido por:

$$
\mathrm{Ka}=\tan ^{2}\left(45-\frac{\emptyset}{2}\right)
$$

\subsection{Coeficiente de Empuxo Passivo}

Coeficiente de empuxo passivo é o esforço devido a pressão do muro sobre o solo, em razão da deformação do muro no sentido do terrapleno, causando assim uma compressão no solo. É definido por:

$\mathrm{Kp}=\tan ^{2}\left(45+\frac{\emptyset}{2}\right)$

\subsection{Verificação de Fenda de Tração}

Quando há coesão no solo ativo, o estado de tensão que provoca sua ativação, também poderá ocasionar o aparecimento de fendas de tração na superfície. Logo, se existe um solo ativo, coeso e superficial, verifica-se a ocorrência deste fenômeno calculando a profundidade Zo através da fórmula:

$Z_{0}=\frac{-\mathrm{q} \times \mathrm{Ka} 1+2 \times \mathrm{C} 1 \times \sqrt{\mathrm{Ka} 1}}{\gamma 1 \times k a 1}$

\subsection{Cálculo dos Empuxos}

Entende-se pelo cálculo das forças horizontais que interagem com maciço, atuando paralelamente com a superfície do terreno. Os empuxos atuantes no maciço considerando os materiais contidos no projeto executivo estão representados na figura 5: 
Figura 5. Empuxos atuantes considerando materiais especificados em projeto.

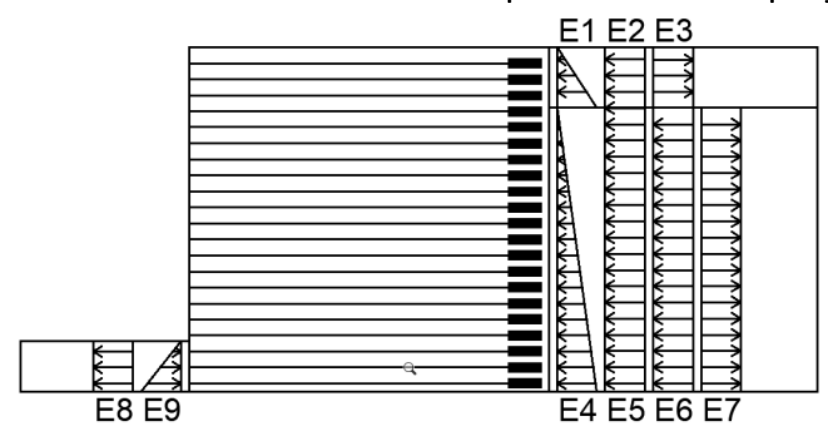

Fonte: Os autores.

Considerando a camada superior do aterro como solo 1 , a camada inferior do aterro como solo 2 e a camada de solo passiva como solo 3 , pode-se ler:

- $\quad$ E1 $\rightarrow$ Peso Próprio do solo 1:

$$
\mathrm{E} 1=\frac{\gamma 1 \times \mathrm{Ka} 1 \times \mathrm{h} 1^{2}}{2}
$$

- $\quad$ E2 $\rightarrow$ Carregamento (q) no solo 1:

$\mathrm{E} 2=\mathrm{q} \times \mathrm{h} 1 \mathrm{xKa} 1$

- $\quad$ E3 $\rightarrow$ Coesão do Solo 1:

$\mathrm{E} 3=-2 \times \mathrm{C} 1 \times \mathrm{h} 1 \times \sqrt{\mathrm{Ka} 1}$

- E4 $\rightarrow$ Peso Próprio do solo 2:

$\mathrm{E} 4=\frac{\gamma 2 \times \mathrm{Ka} 2 \times \mathrm{h} 2^{2}}{2}$

- $\quad$ E5 $\rightarrow$ Sobre Carga do solo 1:

$\mathrm{E} 5=(\mathrm{h} 1 \times \gamma 1) \times \mathrm{h} 2 \times \mathrm{Ka} 2$
- $\quad$ E6 $\rightarrow$ Carregamento (q) no 2:

$\mathrm{E} 6=\mathrm{q} \times \mathrm{h} 2 \times \mathrm{Ka} 2$

- $\quad$ E7 $\rightarrow$ Coesão do Solo 2:

$\mathrm{E} 7=-2 \times \mathrm{C} 2 \times \mathrm{h} 2 \times \sqrt{\mathrm{Ka} 2}$

- $\quad$ E8 $\rightarrow$ Coesão do Solo 3:

$\mathrm{E} 8=-2 \times \mathrm{C} 3 \times \mathrm{h} 3 \times \sqrt{\mathrm{Kp} 3}$

- $\quad$ E9 $\rightarrow$ Peso Próprio do solo 3:

$\mathrm{E} 9=\frac{\gamma 3 \times \mathrm{Kp} 3 \times \mathrm{h} 3^{2}}{2}$

Já os empuxos atuantes no maciço considerando o material utilizado na execução do muro estão representados na figura 6 :

Figura 6. Empuxos atuantes considerando material utilizado na execução (BGS).

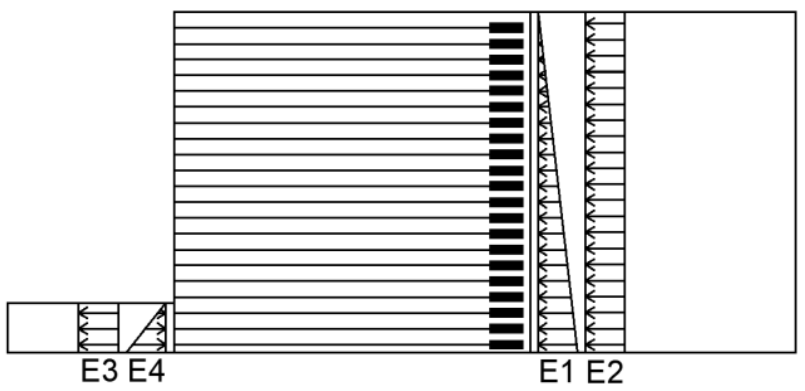

Fonte: Os autores. 
Que são:

- $\quad$ E1 $\rightarrow$ Peso Próprio do BGS:

$\mathrm{E} 1=\frac{\gamma \mathrm{GBS} \times \mathrm{KaBGS} \times \mathrm{hBGS}^{2}}{2}$

- $\quad$ E2 $\rightarrow$ Carregamento (q) no BGS:

$\mathrm{E} 2=\mathrm{q} \times \mathrm{hBGS} \times \mathrm{KaBGS}$

- $\quad$ E3 $\rightarrow$ Coesão do Solo 3:

$\mathrm{E} 3=-2 \times \mathrm{C} 3 \times \mathrm{h} 3 \times \sqrt{\mathrm{Kp} 3}$

- E4 $\rightarrow$ Peso Próprio do solo 3:

$\mathrm{E} 4=\frac{\gamma 3 \times \mathrm{Kp} 3 \times \mathrm{h} 3^{2}}{2}$

\subsection{Força Normal Efetiva na Estrutura ( $N^{\prime}$ )}

É a força efetiva que age no maciço no sentido vertical, causada pelo peso da estrutura, que é descrita por:

$\mathrm{P}=\mathrm{b} \times \mathrm{h} \times \gamma \mathrm{BGS}$

\subsection{Fator de Segurança ao Deslizamento}

A segurança contra o deslizamento da estrutura de contenção consiste na verificação do equilíbrio das componentes horizontais das forças atuantes, com a aplicação de um fator de segurança adequado, este é descrito por:

$\mathrm{Fs}_{\mathrm{d}}=\frac{\Sigma \mathrm{Ep}+(\mathrm{Cax} \mathrm{b})+\mathrm{N}^{\prime} \mathrm{x} \tan \emptyset}{\Sigma \mathrm{Ea}} \geq 1,5$

\subsection{Fator de Segurança ao Tombamento}

Para que o muro não tombe em torno da extremidade externa, o momento resistente deve ser maior do que o momento solicitante. Deste modo, o $\mathrm{Fs}_{\mathrm{T}}$ é definido por:

$\mathrm{FS}_{\mathrm{T}}=\frac{\Sigma \mathrm{MR}}{\Sigma \mathrm{MA}} \geq 1,5$

Sendo:

- $\quad \Sigma M R=$ Somatório dos Momentos Resultantes;

- $\quad \Sigma M A=$ Somatório dos Momentos Resultantes

\section{RESULTADOS}

Para os cálculos realizados considerando os materiais contidos no projeto executivo da estrutura de contenção, foram utilizados os dados elencados na tabela 1 abaixo, bem como resultados obtidos para fator de segurança ao deslizamento $\left(F s_{d}\right)$ e fator de segurança ao tombamento $\left(F s_{T}\right)$ : 
Tabela 1. Dados do Muro Projetado

\begin{tabular}{c|c}
\hline \multicolumn{2}{c}{ Muro Projetado } \\
\hline ү Solo $1\left(\mathrm{kN} / \mathrm{m}^{3}\right)$ & 17,0 \\
S Solo $2\left(\mathrm{kN} / \mathrm{m}^{3}\right)$ & 17,0 \\
Coesão Solo 1 $\left(\mathrm{kN} / \mathrm{m}^{2}\right)$ & 20,0 \\
Coesão Solo 2 $\left(\mathrm{kN} / \mathrm{m}^{2}\right)$ & 5,0 \\
$\varnothing$ Solo 1 $\left(^{\circ}\right)$ & 18,0 \\
$\varnothing$ Solo 2 $\left(^{\circ}\right)$ & 30,0 \\
Ka1 & 0,528 \\
Ka2 & 0,333 \\
Kp3 & 3,0 \\
$\Sigma$ Empuxo Ativo & 191,63 \\
$(\mathrm{kN} / \mathrm{m})$ & 18,19 \\
$\Sigma$ Empuxo Passivo & 1315,80 \\
$(\mathrm{kN} / \mathrm{m})$ & $\mathbf{4 , 2}$ \\
$\mathrm{N}^{\prime}(\mathrm{KN} / \mathrm{m})$ & $\mathbf{6 , 9}$ \\
\hline FSd & \\
\hline FSt &
\end{tabular}

$F s_{d}=4,2>1,5 \therefore 0 \mathrm{k}$

$F s_{t}=6,9>1,5 \therefore \mathrm{Ok}$

Para os cálculos realizados considerando o material do qual foi executada a estrutura de contenção (BGS), foram utilizados os dados elencados na

Tabela 2. Dados do Muro Executado

\begin{tabular}{c|c}
\hline \multicolumn{2}{c}{ Muro Executado } \\
\hline ү BGS $\left(\mathrm{kN} / \mathrm{m}^{3}\right)$ & 23,0 \\
Coesão BGS $\left(\mathrm{kN} / \mathrm{m}^{2}\right)$ & 0,0 \\
Coesão Base $\left(\mathrm{kN} / \mathrm{m}^{2}\right)$ & 5,0 \\
$\varnothing$ BGS $\left(^{\circ}\right)$ & 35,0 \\
$\varnothing$ Base $\left(^{\circ}\right)$ & 30,0 \\
Ka1 & 0,271 \\
Kp2 & 3,0 \\
$\Sigma$ Empuxo Ativo & 277,11 \\
$(\mathrm{kN} / \mathrm{m})$ & 18,19 \\
$\Sigma$ Empuxo Passivo & 1780,20 \\
$(\mathrm{kN} / \mathrm{m})$ & $\mathbf{3 , 9}$ \\
$\mathrm{N}^{\prime}$ (KN/m) & $\mathbf{9 , 2}$ \\
\hline FSd &
\end{tabular}


$F s_{d}=3,9>1,5 \therefore \mathrm{Ok}$

$F s_{t}=9,2>1,5 \therefore \mathrm{Ok}$

\section{CONCLUSÃO}

Pode-se observar uma redução no valor do fator de segurança ao deslizamento (FSd) e um aumento no valor do fator de segurança ao tombamento (FSt), variações essas devidas à alguns fatores, como, as diferentes massas específicas dos materiais utilizados, resultando em diferentes pesos próprios dos muros analisados, diferentes valores de empuxos ativos, dentre outros fatores.

Tais variações não caracterizam uma proeminente falha da estrutura analisada, no que diz respeito exclusivamente aos dois fatores de segurança analisados, uma vez que mesmo com alterações, os fatores de segurança se encontram dentro no mínimo exigido para este tipo de estrutura.

Por outro lado, devido à mudança de material, pode-se observar um aumento relativamente acentuado no peso próprio da estrutura, podendo levar à ruptura, seja por puncionamento ou generalizada, do solo da base do muro. Tal falha pode acarretar recalques diferenciais ou globais causando danos à rodovia locada em cima da estrutura de contenção.

Portanto, para assegurar que as alterações feitas na estrutura não venham a causar futuras falhas, deve-se analisar o projeto como um todo, pois mesmo não caracterizando falhas por deslizamento e tombamento da estrutura, analisadas por este trabalho, a mesma pode vir a sofrer com patologias ocasionadas pelas não conformidades geradas, como aumento do peso específico do aterro, instabilidades internas no maciço, causadas pela falta de coesão real no material utilizado, deformações causadas pela acomodação das partículas do material utilizado no aterro, tendo em vista o transito intenso de veículos pesados logo acima do mesmo, dentre outras.

\section{REFERÊNCIAS}

CORREIA, F. S., Júnior, J. F., DIAS, M. C.; LIMA, $S$. F. Análise dos principais problemas construtivos decorrentes de falhas de projeto. Cadernos de Graduação - Ciências exatas e tecnológicas, nov., 2017.

MAGALHÃES , T. A.; AZEVEDO , C. P. Análise técnica e econômica de estruturas de contenção de taludes. COBRAMSEG out., 2016 .

MARCELLI, M. (2007). Sinistros na construção civil - causas e soluções para danos e prejuizos em obras. São Paulo: PINI, 2007. 270 p..

MAYR, L. R. Falhas de projeto e erros de execução: Uma Questão de Comunicação. Engenharia de Produção-UFSC, 2000.

MITCHELL , J. K.; VILLET , W. C. (June de 1987). Reinforcement of earth slopes and embankments. National Cooperative Highway Reserch Program Report.

SILVA, N. H. Muros de terra armada verificação da segurança. Dissertação (Mestrado em Engenharia Civil) - Ramo de Estruturas e Geotecnia - Universidade Nova de Lisboa, 2012. 